\title{
Origin of contrast in STM images of oxygen on Pd(111) and its dependence on tip structure and tunneling parameters
}

\author{
Jose Manuel Blanco, ${ }^{1}$ Cesar González, ${ }^{1}$ Pavel Jelínek,,${ }^{1,2}$ José Ortega, ${ }^{1}$ Fernando Flores, ${ }^{1}$ Rubén Pérez, ${ }^{1}$ Mark Rose, ${ }^{3}$ \\ Miquel Salmeron, ${ }^{3}$ Javier Méndez, ${ }^{4,5}$ Joost Wintterlin, ${ }^{4}$ and Gerhard Ertt ${ }^{4}$ \\ ${ }^{1}$ Departamento de Física Teórica de la Materia Condensada, Universidad Autónoma de Madrid, E-28049 Madrid, Spain \\ ${ }^{2}$ Institute of Physics, Academy of Sciences of the Czech Republic, Cukrovarnická 10, 1862 53, Prague, Czech Republic \\ ${ }^{3}$ Institute of Physics, University of California, Berkeley, California, USA \\ ${ }^{4}$ Fritz-Haber Institute der MPG, Berlin, Germany \\ ${ }^{5}$ Instituto de Ciencia de Materiales de Madrid, ICMM-CSIC, Madrid 28049, Spain
}

(Received 25 June 2004; published 10 March 2005)

\begin{abstract}
The dependence of the contrast and symmetry of scanning tunneling microscope images of $\mathrm{O} / \mathrm{Pd}(111)-2$ $\times 2$ on the structure of the tunneling tip and on tunneling parameters is explained using first-principles density functional theory. Experimentally, the contrast changes in different ways when a metal-terminated tip over hcp and top sites changes its bias and tip-sample distance. These changes are also reflected in the symmetry of the image. A detailed analysis of the tunneling contributions indicates that for the metallic tips, the Pd $d$ orbitals are determining the image symmetry at close range and low bias, while at larger separations and high bias the $\mathrm{Pd} p_{z}$ orbitals are the ones that control the image contrast. For oxygen-terminated tips, we predict a positive image contrast, associated with the tip oxygen bonds, as opposed to the negative contrast images obtained with metallic tips.
\end{abstract}

DOI: 10.1103/PhysRevB.71.113402

PACS number(s): 68.37.Ef, 71.15.Mb, 73.40.Gk

The study of the atomic structure of surfaces and their relation with chemical and physical properties is one of the most active areas of research in the materials sciences. ${ }^{1}$ Understanding the nature of the adsorption sites of atoms and molecules and their dissociation provides insight into the basic steps of generally complex catalytic processes. Scanning tunneling microscopy (STM) plays a key role in the characterization of the adsorption of molecules as it provides unparalleled detail of the structure of the adsorption site on ideally clean metal surfaces or in the proximity of defects. ${ }^{2}$

Because the tunneling process involves wave functions associated with specific electronic orbitals, it is clear that the image contrast depends on the surface and tip electronic structure. In this work, we analyze experimentally and theoretically the structure of STM images of atomic oxygen adsorbed on $\mathrm{Pd}(111)$, which form a $2 \times 2$ periodic structure at low coverage. As we shall see, proper consideration of the tunneling parameters, including applied bias and tip-sample distance, as well as tip composition, allows us to understand the contributions to the image contrast of various tip and surface orbitals, which is crucial for a correct interpretation of STM images.

Figure 1 summarizes the results of two different experiments addressing the same $\mathrm{O} / \mathrm{Pd}(111)-2 \times 2$ system. In both experiments, the $\mathrm{Pd}$ crystal was cleaned by cycles of ion-sputtering and annealing. In the first case, the atomic oxygen layer was prepared by thermal dissociation of a layer of adsorbed molecules from 60 to $160 \mathrm{~K}$, which produced patches or islands with internal $(2 \times 2)$ ordering. Large, ordered areas of the same structure were produced in the other set of experiments by exposure to molecular oxygen gas at room temperature. In both cases, STM images were acquired at low temperature. The image of Fig. 1(a) was obtained with a Pt tip and low bias $(V=-0.059 V$ and $I=16.9 \mathrm{nA})$, while the data of Fig. 1(b) correspond to an Ir tip and a larger bias $(V=-1.4 V$ and $I=0.7 \mathrm{nA})$.

The crystallographic orientation of the surface was determined from images showing the atomic corrugation in two consecutive terraces separated by monoatomic steps. In this manner, the low coverage image presented in Fig. 1(a), where both the oxygen and surrounding metal atoms are imaged, can be interpreted in terms of oxygen atoms adsorbed on hollow fcc sites and producing a depression. These same interpretation can be applied to the denser arrays shown in Fig. 1(b), where the oxygen atoms are responsible for the dark areas in the image. However, apart from this basic feature, common to most of the experimental images (cases in which $\mathrm{O}$ atoms are imaged as protrusions are also observed and will be discussed below), the shape of the depression associated to the oxygen atom in both topographs is substantially different: triangular in Fig. 1(a) and circular in Fig. 1(b). The differences in the contrast structure of the two images are manifested more quantitatively in the line scans shown as insets in Fig. 1, taken along the same crystallographic direction. As can be seen, the maxima in the images (brighter spots) are associated with either top or hollow hcp positions, showing that the naive identification of protrusions with atomic positions is not always justified. Relevant to the present discussion is the shift of the contrast maximum from the top site, near the center of the scan line in (a) at low bias, to the hcp hollow site in the case of the high bias image shown in (b).

This change in image contrast and symmetry calls for a first-principles analysis that goes beyond the standard Tersoff-Hamman approach. ${ }^{3}$ In this paper, we combine standard density functional theory (DFT) plane-wave (PW) pseudopotential methods for the accurate determination of the tip and sample structure and electronic properties, with a 


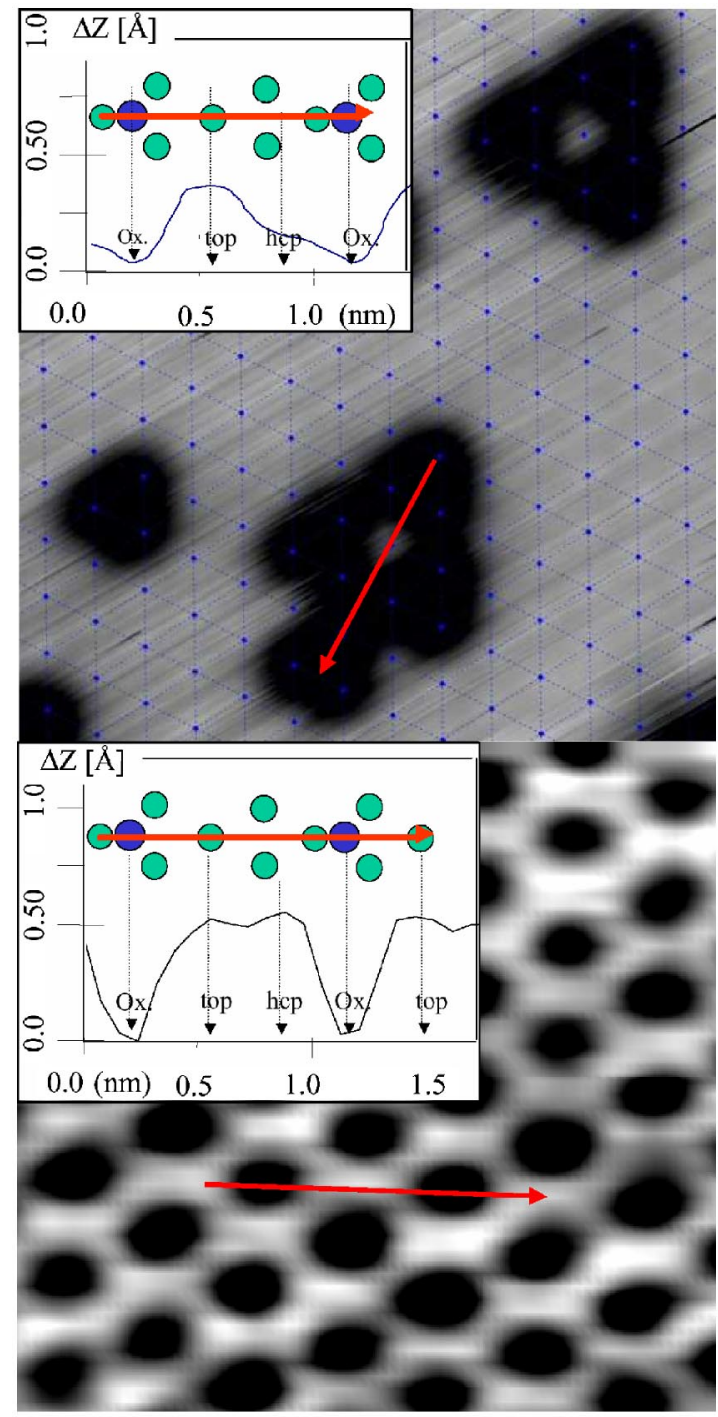

FIG. 1. (Color online) STM images for O adsorbed on Pd(111) forming a $2 \times 2$ superstructure. (a) Low coverage limit measured with a Pt tip, $V=-0.059 \mathrm{~V}$ and $I=16.0 \mathrm{nA}$; the underlying metal surface is also shown. (b) Monolayer coverage measured with an $\mathrm{Ir}$ tip, $V=-1.4 \mathrm{~V}, I=0.7 \mathrm{nA}$.

calculation of STM currents based on nonequilibrium Keldysh-Green function techniques. ${ }^{4-6}$ The connection between the structural and transport calculations is done through a fast local-orbital DFT technique (Fireball2003), ${ }^{7,8}$ which efficiently maps the electronic Hamiltonian determined from the PW calculation, and can be naturally linked with the transport formalism, which is expressed in terms of localized orbitals. ${ }^{6}$ This approach incorporates the influence of the bias and current conditions as well as a realistic description of the tips chemical nature (either Pt or Ir) used in the experiments and their possible contamination.

In our approach, ${ }^{5,6}$ the electrical current flowing through the tip-sample system, (assumed to be described in an orthogonal local orbital basis) in the limit of small $T_{t s}$ that is relevant to the typical tip-sample distances in the experiments, is given by

$$
\begin{aligned}
I=\sum_{i, j} I_{i j}= & \sum_{i, j} \frac{4 \pi e}{\hbar} \int_{-\infty}^{\infty} d \omega\left[f_{t}(\omega-e V)-f_{s}(\omega)\right]\left(\hat{\rho}_{s s}\right)_{i j}(\omega) \\
& \times\left[\sum_{p, q}\left(\hat{T}_{s t}\right)_{j p}\left(\hat{\rho}_{t t}\right)_{p q}(\omega-e V)\left(\hat{T}_{t s}\right)_{q i}\right]
\end{aligned}
$$

where $V$ is the bias voltage, $f_{i}(\omega)(i=t, s)$ is the corresponding Fermi-Dirac distribution function, $\hat{T}_{t s}$ are the corresponding matrix elements linking the basis orbitals in the tip and sample, and $\hat{\rho}_{t t}$ and $\hat{\rho}_{s s}$ are the respective density of states matrices for the isolated tip and sample $\left(\hat{T}_{t s}=0\right)$. $I_{i j}$ represents the tunneling current associated with the sample $(i j)$ bond; for $i=j, I_{i i}$ represents the current through the $i$ orbital. Similarly, we can write $I=\Sigma_{p q} I_{p q}, I_{p q}$ being the current associated with the tip $(p q)$ bond.

The relaxed structure and electronic properties of the isolated tip and sample have been determined using CASTEP, ${ }^{9}$ a first-principles total energy DFT generalized gradient approximation pseudopotential plane-wave code. ${ }^{10}$ In the $\mathrm{O} / \mathrm{Pd}(111)-2 \times 2$ relaxed geometry, the three $\mathrm{Pd}$ atoms close to the $\mathrm{O}$ have in-plane and upwards movements of $0.05 \AA$, the fourth Pd atom on the unit cell moves downwards $0.02 \AA$ (with respect to their positions in the clean surface) and the $\mathrm{O}$ stands $1.12 \AA$ above the surface, in agreement with previous calculations. ${ }^{11}$

The tip apex was simulated by a tetrahedral four-atom cluster coupled to the (111) slab of the corresponding bulk material (Pt or Ir). Tip chemical composition was simulated with either a pure Pd tip or by replacing the apex Pd atom by O. Details on the relaxed structures of the different tips will be published elsewhere. ${ }^{12}$

As mentioned above and as discussed in detail in Ref. 6, the connection between the structural and transport calculations is done through a fast local-orbital DFT technique (Fireball2003). ${ }^{7,8}$ In Fireball, the valence wave functions for either $\mathrm{O}$ or the transition metal atoms are expanded in a set of strictly localized pseudoatomic orbitals. This offers a very favorable accuracy/efficiency balance if the atomic-like basis set is chosen carefully (see Refs. 13-15).

The hopping elements $\left(T_{t s}\right)_{p i}$ have been calculated using a dimer approximation, whereby each atom is described by pseudoatomic orbitals with a cutoff radius larger than $10 \AA$, thus reproducing the behavior of the real free-atom orbitals. It is shown in Ref. 6 that this prescription yields accurate tunneling currents for long tip-sample distances, which is the case that corresponds to the present study.

In Fig. 2(a) we show calculated $\mathrm{O} / \mathrm{Pd}(111)-(2 \times 2)$ constant current STM images for a tetrahedral Pt tip, a negative bias of $60 \mathrm{meV}$, and a tip-sample current of $58 \mathrm{nA}$ [giving a contact resistance of $\sim 10^{6} \Omega$, in agreement with Fig. 1(a) conditions]. The inset of Fig. 2(a) shows the corrugation along the relevant scan line. The good agreement between our calculations and the experimental data in Fig. 1(a) is evident. The shape of the STM image, which depends critically on the relative corrugation heights of the top and hcp positions along the scan line, is also correctly predicted. The main result is that when the corrugation height of the top position is higher than that of the hcp site a triangular shape 


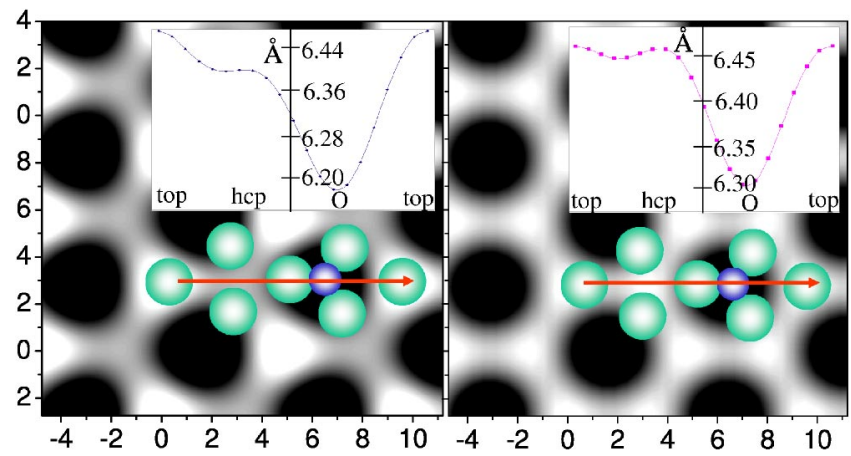

FIG. 2. (Color online) Calculated $\mathrm{O} / \mathrm{Pd}(111)-2 \times 2 \mathrm{STM}$ images for (a) Pt tip, $V=-0.060 \mathrm{~V}$ and $I=58 \mathrm{nA}$; (b) Ir tip, $V$ $=-1.0 \mathrm{~V}$ and $I=158 \mathrm{nA}$.

is obtained, as in Fig. 2(a); when both points have the same corrugation the shape of the oxygen depression becomes circular [STM images calculated for a Ir tip, $V=-1.0 \mathrm{~V}$ (positive) and $I=158 \mathrm{nA}$, Fig. 2(b)]. Finally, when the corrugation at the hcp site is higher than that over the top site, the triangular shape reappears but inverted relative to the one of Fig. 2(a). ${ }^{16}$

These observations allow us to analyze all the $\mathrm{O} / \mathrm{Pd}(111)-(2 \times 2)$ STM images characterized by a depression at the position of the $\mathrm{O}$ atoms and the triangular or circular shapes around them in terms of the current profiles along the scan line.

Figure 3(a) shows different components of the tunneling current calculated along the scan line for a constant tipsample distance of $6.45 \AA$ defined as the distance between the centers of the apex atom and the last $\operatorname{Pd}(111)$ layer and corresponds to the top position of the inset of Fig. 2(a). The magnitude of the total current is basically given by the current through $\mathrm{Pd}-\mathrm{Pd}$ orbitals. The role of the $\mathrm{O}$ orbitals is basically to create a large depression in the STM current on top of the $\mathrm{O}$ atoms. The bonding between the $\operatorname{Pd} p_{z}$ and the $\mathrm{O} p_{z}$ orbitals yields the largest contribution to this image depression. ${ }^{17}$ On the other hand, as mentioned above, the shape of the image around the $\mathrm{O}$ is determined by the relative intensities for the top and hcp positions. Figure 3(a) shows that the currents through the $\mathrm{O}$ orbitals are the same for both sites. The currents through the $\mathrm{Pd}(d-s$ or $d-p)$ bonds however, are responsible for the shape, round or triangular of the $\mathrm{O}$ depression, indicating that the symmetry of the STM image depends crucially on the $\operatorname{Pd} d$ density of states. This finding also explains the dependence of STM images with the tip-sample distance (not shown experimentally in this paper): for large distances, the contribution of the $\operatorname{Pd} d$ orbitals to the tunneling current decreases more rapidly than the contribution from the more extended $s$ and $p$ orbitals and tend to make the $\mathrm{Pd}(d-s$ or $d-p)$ orbital contributions to the current negligible. This explains why the shape of the $\mathrm{STM}$ image around $\mathrm{O}$ for large distances is a circle.

A full understanding of the STM image dependence on the applied bias [Fig. 2(b)] can be obtained by analyzing the current components $I_{i j}$, as shown in Fig. 3(b). Comparing Figs. 3(a) and 3(b), we see that the main effect of the applied bias is to change the shape of the current profile associated with the $\operatorname{Pd}(d-p$ or $d-s)$ bonds: this component is now very flat, yielding a total STM current with similar values at the top and hep sites. ${ }^{18}$ As discussed below, this behavior is independent of the tip metal atom, Pt or Ir.

Finally, we analyzed how the STM images depend on the chemical nature of the tip. First of all, we find that different metal atoms at the tip apex do not change the simulated images appreciably. Indeed, we obtain nearly identical tunneling currents with Pd and Pt tips, as shown in Fig. 4(a). Nonmetallic tips, specifically oxygen-terminated tips, were constructed by replacing the $\mathrm{Pd}$ atom at the tip apex by an $\mathrm{O}$ atom. For the metal-terminated tips, the tunneling current is associated mainly with the apex atom [Fig. 4(a)] and in particular, with its $p_{z}$ orbital. This explains why different metal atoms yield similar images and why tips of the same metal, but having different geometries, may have quite different tunneling currents due to changes in the $p_{z}$ density of states. However, oxygen contamination of the tip introduces significant qualitative changes [see Fig. 4(b)]: oxygen atoms are now imaged as bright protrusions located on top of the atom (images for this case, in agreement with this result, will be published elsewhere ${ }^{19}$ ).

These changes can be easily understood in terms of the $I_{p q}$ current components: while for the metal tip most of the current is associated with the apex atom, for the oxygenterminated tip, the apex atom contributes a very small fraction, and most of the current goes through the three metal atoms on the layer above the oxygen, a result in agreement with previous approximate calculations using a scattering formalism and the extended Debye-Huckel approximation ${ }^{20}$ ).

In conclusion, based on a DFT approach, we have shown
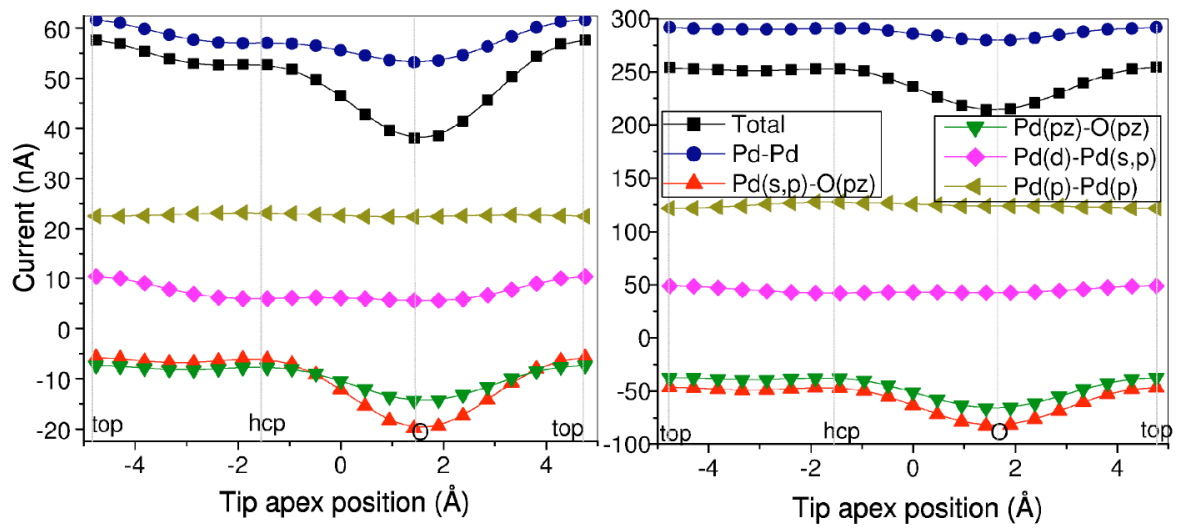

FIG. 3. (Color online) Different components of the total tunneling current along the top-top scan line [see Fig. 2] for (a) $d$ $=6.45 \AA, V=-0.060 \mathrm{~V}$ and Pt tip; (b) $d=6.45 \AA, V=-1.0 \mathrm{~V}$ and $\mathrm{Ir}$ tip. 


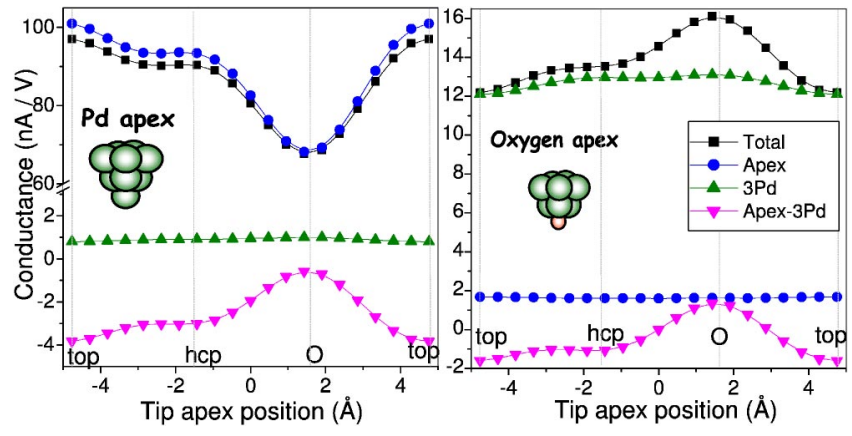

FIG. 4. (Color online) Different components of the tip-sample conductance as a function of the tip position along the scan line (see Fig. 2) with a tip-sample distance of $6.45 \AA$, for (a) a Pd tip and (b) an O-contaminated tip.

that the contrast and symmetry of STM images of $\mathrm{O} / \mathrm{Pd}(111)-(2 \times 2)$ that are found experimentally to vary from a triangular to a circular depression, ${ }^{21}$ or even to a protrusion when the tip is contaminated with $\mathrm{O}$, can be explained by the relative contributions of short range $\mathrm{Pd} d$ orbitals and long range $\mathrm{Pd} s$ and $\mathrm{Pd} p$ orbitals. The former are accessed at short tip-surface distances and low bias voltages.
The latter are dominant at large separations and/or larger bias voltages. This behavior could be understood with the use of first-principles density functional calculations combined with a Keldysh-Green function formalism. The results also show that for a clean tip, the sample $\mathrm{O} p_{z}$ orbital is mainly responsible of the image depression at the oxygen position.

All these results conclusively prove that the shape and symmetry of the image (triangular or circular), depend crucially on the contribution to the tunneling current of the $\mathrm{Pd} d$ orbitals that can be reduced or increased by changing the tip-sample distance or the applied bias. Additionally, we have also shown that the protrusion found on top of oxygen for an oxygen contaminated tip is associated mainly with the tip $\mathrm{Pd}-\mathrm{O}$ bonds.

P. J. gratefully acknowledges financial support by the Spanish Ministerio de Educacion, Cultura y Deportes. This work has been supported by the DGI-MCyT (Spain) under Contract Nos. MAT2001-0665, MAT2002-01534, and MAT2002-00395. M. S was supported by the Director, Office of Energy Research, Office of Basic Energy Sciences, Materials Science Division, of the U.S. Department of Energy under Contract No. DE-AC03-76F00098. Computer time at the CCC-UAM is gratefully acknowledged.
${ }^{1}$ See, for instance, Impact of Surface Science in Catalysis, edited by B. C. Gates and H. Knozinger, in Adv. Cat. Vol. 45 (Academic, New York, 2000).

${ }^{2}$ T. Mitsui, M. Rose, E. Fomin, F. Ogletree, and M. Salmeron, Surf. Sci. 511, 259 (2002); M. K. Rose, A. Borg, J. C. Dunphy, T. Mitsui, D. F. Ogletree, and M. Salmeron, ibid. 547, 162 (2003); J. Wintterlin, R. Schuster, and G. Ertl, Phys. Rev. Lett. 77, 123 (1996).

${ }^{3}$ J. Tersoff and D. R. Hamann, Phys. Rev. B 31, 805 (1985).

${ }^{4}$ L. V. Keldysh, Sov. Phys. JETP 20, 1018 (1965).

${ }^{5}$ N. Mingo, L. Jurczyszyn, F. J. Garcia-Vidal, R. Saiz-Pardo, P. L. de Andres, F. Flores, S. Y. Wu, and W. More, Phys. Rev. B 54, 2225 (1996).

${ }^{6}$ J. M. Blanco, C. Gonzalez, P. Jelinek, J. Ortega, F. Flores, and R. Perez, Phys. Rev. B 70, 085405 (2004).

${ }^{7}$ J. P. Lewis, K. R. Glaesemann, G. A. Voth, J. Fritsch, A. A. Demkov, J. Ortega, and O. F. Sankey, Phys. Rev. B 64, 195103 (2001).

${ }^{8}$ P. Jelinek et al., cond-mat/0409509 (unpublished).

${ }^{9}$ CASTEP 4.2 Academic version, licensed under the UKCP-MSI Agreement, 1999.

${ }^{10} \mathrm{Pd}$ and $\mathrm{O}$ atoms are described with ultrasoft pseudopotentials and wave functions expanded with a PW cutoff of $340 \mathrm{eV}$. For the integration on the two-dimensional first Brillouin zone, we con- sider a mesh of 16 Monkhorst-Pack $k$ points and a smearing width of $\sigma=0.2 \mathrm{eV}$.

${ }^{11}$ A. Seitsonen et al., Surf. Sci. 468, 176 (2000).

${ }^{12} \mathrm{~J}$. M. Blanco et al. (unpublished).

${ }^{13}$ J. Ortega, Comput. Mater. Sci. 12, 192 (1998).

${ }^{14}$ J. Ortega, R. Perez, and F. Flores, J. Phys.: Condens. Matter 12, L21 (2000).

${ }^{15}$ P. Jelinek, R. Pérez, J. Ortega, and F. Flores, Phys. Rev. B 68, 085403 (2003).

${ }^{16}$ J. Méndez, S. H. Kim, J. Cerdá, J. Wintterlin, and G. Ertl, Phys. Rev. B 71, 085409 (2005).

${ }^{17}$ Although the $\operatorname{Pd} p$ orbitals have a small occupancy their density of states at $E_{f}$ is large enough to yield a dominant contribution to the tunneling current.

${ }^{18} \mathrm{We}$ have found similar effects for a positive polarity: $V=1.0 \mathrm{~V}$ and $d=6.45 \AA$ (details will be published elsewhere).

${ }^{19}$ M. K. Rose, A. Borg, J. C. Dunphy, T. Mitsui, D. F. Ogletree, and M. Salmeron, Surf. Sci. 561, 69 (2004).

${ }^{20}$ H. C. Galloway, P. Sautet, and M. Salmeron, Phys. Rev. B 54, R11 145 (1996).

${ }^{21}$ A recently published work has presented a similar study for the $\mathrm{Ru}(0001)-\mathrm{O}(2 \times 2)$ system: F. Calleja et al., Phys. Rev. Lett. 92, 206101 (2004). 